\title{
HUCHA CERÁMICA DEL S. III D.C. DEL MUSEO ARQUEOLÓGICO MUNICIPAL DE LORCA (MURCIA) ${ }^{1}$
}

\author{
ISIDRO MARTÍNEZ MIRA
}

Universidad de Alicante

\begin{abstract}
Con este artículo queremos realizar una serie de comentarios sobre un objeto singular conservado en el Museo Arqueológico Municipal de Lorca (Murcia): una hucha cerámica romana del siglo III d.C.
\end{abstract}

In this article, we discuss an unusual piece kept at the Museo Arqueológico Municipal of Lorca (Murcia): a Roman terracotta moneybox dating to the 3rd century A.D.

\section{INTRODUCCIÓN}

Normalmente el hallazgo de depósitos o conjuntos numismáticos viene asociado, en la mayoría de los casos, a los restos del recipiente que en su momento los contuvo. Estos recipientes, para los depósitos hallados en la Península Ibérica, con una cronología del siglo III d.C., presentan una variada tipología en cuanto a su naturaleza y su forma (Martínez Mira, 1995-97, 153):

-Cerámicos: ánforas, platos, vasos, jarras, ollas, etc.

-Metálicos de hierro, cobre o bronce, plata, etc: calderos, ollas, vasos.

-Vidrio: ampollas.

- Madera: cajitas.

-Material orgánico: cuero, lino, tela.

Cada recipiente se caracteriza por estar adaptado a la finalidad del depósito monetario que contiene, habitualmente, los contenedores hechos de material orgánico se corresponden en su mayoría con monederos que suelen contener unas pocas de las monedas circulantes en su ámbito geográfico, en el momento de su pérdida. Por el contrario, los hallazgos contenidos en recipientes de mayor solidez en su estructura y también de mayor capacidad, normalmente responden a otros parámetros en su formación y su contenido no suele ser el reflejo de la moneda circulante en un determinado momento y lugar sino el de una acumulación realizada durante un lapso determinado de tiempo e incluso pueden mostrarnos una se- lección de las piezas que lo componen de entre los valores circulantes.

El mayor número de recipientes conservados son los de tipo cerámico, de carácter doméstico, adaptados a la función de contener un determinado grupo de monedas que, por lo general, se corresponden con la renta familiar disponible. Su hallazgo se suele realizar en lugares más o menos resguardados pero, casi siempre, pertenecientes a ámbitos domésticos.

En el caso que nos ocupa, estamos ante esta última situación, con la diferencia de que solo disponemos de una moneda del conjunto monetario que presumiblemente contuvo en su interior.

En la actualidad todavía se desconoce cuál era la denominación que los romanos daban a las huchas. El término loculus designa a un pequeño espacio reservado para un objeto y el modo loculi, en plural designaría a cualquier recipiente monetario y entre ellos a las huchas (Thedenat, 1969, 1293) aunque también podria ser que se usase el término Thesauros (Robinson, 1924, 239). Según el Diccionario de la Lengua Española de la Real Academia Española, la palabra hucha designa una alcancía de barro o caja de madera o metal con una sola hendidura, que sirve para guardar dinero (Real Academia Española, 1998, 1128, hucha). Su

\footnotetext{
${ }^{1}$ Queremos agradecer a M. Martínez Rodríguez y J. Ponce García, del Museo Arqueológico Municipal de Lorca (Murcia), las precisiones y facilidades que nos dieron para el estudio de esta pieza.
} 
uso debió de ser muy popular y estar bastante extendido como lo prueba que ya en 1924 se hubieran identificado 50 ejemplares (Robinson, $1924,239)$. Desde entonces su número no ha dejado de crecer ${ }^{2}$ aunque desconocemos si se ha realizado una actualización de su inventario.

A principios del s. XX; Graeven formuló la primera y todavía aún vigente clasificación tipológica de las huchas cerámicas en el mundo romano (Graeven, 1901, 160-189). Básicamente las clasificó en cuatro tipos:

-Tipo I o tipo arca: Su forma es la de un pequeño cofre o arca. Al menos cuatro ejemplares de este tipo hechos con el mismo molde se hallaron en casas de Pompeya. Pueden llevar decoración, la ranura de inserción de las monedas está en la cara superior (Graeven, 1901,168 y fig. 6 y 7). Robinson describe un ejemplar de este tipo, perteneciente a la Universidad de Yale, decorado con un cerdo (símbolo entre los romanos de buena suerte) y con una inscripción: FELIX, (Robinson, 1924, 240, fig. 1)

- Tipo Il o tipo olla: Su forma es el de una olla cerrada por su parte superior, hecha a torno y con una factura tosca, (la forma cerámica más extendida usada para contener monedas en las casas eran las ollas bien con una tapa o con un cuello estrecho). Normalmente no suelen presentar decoración y presentan la ranura de inserción de las monedas en su parte superior de forma horizontal aunque en algún caso también la puede presentar en vertical como sería el caso de la hucha de Lincoln en Inglaterra (White, 1981, 302-305 y fig. 6), Dentro de este grupo se produce una gran variabilidad en las formas dado que son cerámicas de uso común y orígen local. Es la más práctica de los cuatro tipos, quizás por ello sea la forma de hucha que ha perdurado hasta la actualidad (Graeven, 1901, 170; Robinson, 1924, 240-41 y fig. 2)

Los ejemplares de los dos siguientes tipos parecen restringir sus hallazgos a Roma y alrededores.

-Tipo III o tipo cuerpo de lucerna: La denominación de este tipo de hucha proviene de su clara relación con los fabricantes de lucernas: son de forma circular plana y de pequeñas dimensiones; siendo similar tanto en la forma como en sus dimensiones o su decora- ción a los cuerpos de las lucernas. En su mitad superior presenta un medallón moldeado, situándose la ranura de inserción de las monedas en esta cara superior. Algunas de ellas van firmadas por alfareros fabricantes de lucernas (ej: C. IVN(ius) BIT(us)). En el medallón decorativo suelen aparecer Hércules, Ceres, Hermes, Victoria o Fortuna y parece que se regalaban a los niños con motivo de la festividad del Año Nuevo (Graeven, 1901, 177; Robinson, 1924, 242-44 y fig. 3).

-Tipo IV o tipo panal de abejas: Su tipo se corresponde con una forma omphaloide o de panal de abejas. Generalmente presenta representaciones de un dios (Hermes, Victoria o Fortuna por lo general) dentro de una capilla aunque también pueden llevar otras representaciones (en un ejemplar aparece el vencedor de una carrera de carros). La ranura de inserción de las monedas se sitúa en la parte superior por encima de la decoración. Algunas presentan en su parte posterior la firma del alfarero que las realizó como por ejemplo: AEL(ius) MAX(imus) o BAS(ieni) AVGVR(ini) (Graeven, 1901, 184; Robinson, 1924, 244-47 y fig. 4 y $5)$. Dadas su forma y dimensiones Cheilik (Cheilik, 1963, 70-71) las ha relacionado con los cultos familiares de las casas, relacionando su forma, dimensiones y decoración con algunos huecos que presentan determinados lararios, también se les ha atribuido la función de huchas de recompensa para ganadores de competiciones deportivas como las carreras de caballos (Robinson, 1924, 245).

\section{DESCRIPCIÓN DE LA HUCHA:}

El recipiente es una jarra cerámica de color amarillento, con un pie anular de unos $6,6 \mathrm{~cm}$. de diámetro de su base. Presenta un cuerpo esférico que mide en torno a $16,4 \mathrm{~cm}$. en su parte media y de cuya parte superior arranca un cuello que presenta un diámetro en su parte de final de $4,5 \mathrm{~cm}$. De la parte superior del cuello parte una asa trilobulada que acaba en el tercio superior del cuerpo de la jarra. La altura total de la pieza es de unos 19,6 $\mathrm{cm}$.

\footnotetext{
${ }^{2}$ Como muestra damos la cita de varios trabajos recientes en donde se describen huchas romanas: Hensen, 1998, 3-6; Hussong, Cüppers, 1972, 58, Fig, 7 y Dukat, Saric, 199596, $97-110$
} 

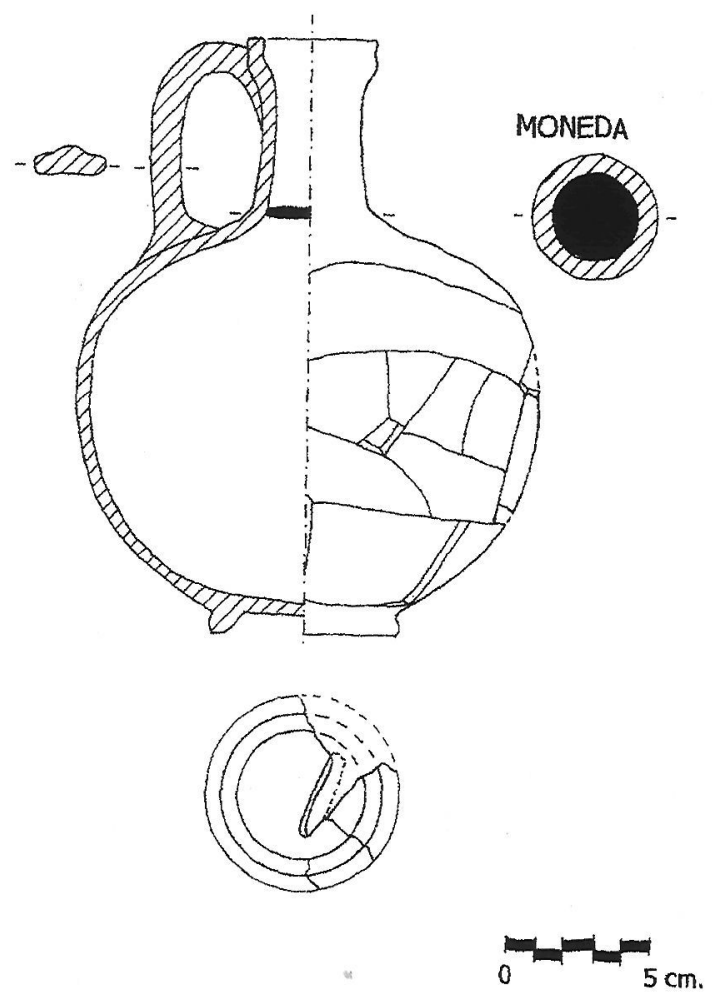

Figura 1: Dibujo de la hucha tomado de Martínez Rodríguez, Ponce Garcia, 1999, 320, fig. 19

El alfarero practicó, antes de su cocción, una incisión longitudinal en la base del pie para poder permitir la introducción de monedas dentro del recipiente. De esta incisión se conservan unos $34 \mathrm{~mm}$ de su longitud original con unos $6 \mathrm{~mm}$. de anchura. Dado que muy probablemente la mayoría de las monedas que contuvo fueran sestercios (aunque muy posiblemente contuviera también radiados), la longitud originaria de esta hendidura debería haber sobrepasado los $42-45 \mathrm{~mm}$. de longitud lo que permitiría la introducción de estas monedas sin ningún problema $y$, por extensión, cualquier moneda de plata o cobre del sistema monetario romano vigente en el período de su utilización.

Del contenido del recipiente solamente se ha conservado una moneda: un sestercio que presenta un reverso del tipo CONCORDIA AVGVSTA. Esta moneda se encuentra encajada tapando el arranque del cuello de la pieza por su parte interna. Los vacíos existentes entre el contorno de la moneda y las paredes internas de la jarra se hallan rellenadas por tierra, procedente del mismo lugar de su hallazgo, que se ha endurecido con el paso del tiempo y que ha ayudado a su fijación. La moneda no ha sido removida de su situación original, por lo que no se ha podido realizar su descripción completa.

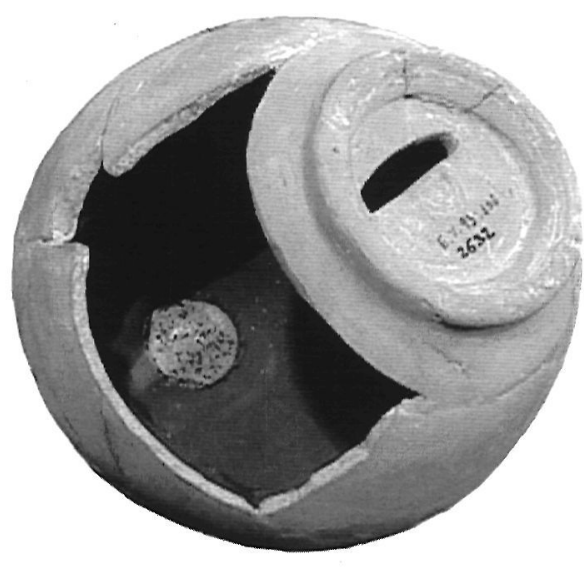

Figura 2: Detalle de la ranura de inserción de las monedas y del sestercio encajado en el interior del arranque del cuello de la hucha

\section{CRONOLOGÍA}

La cronología de un conjunto monetario se suele establecer teniendo en cuenta una serie de variables entre las que destacan:

-las circunstancias arqueológicas de su aparición que abarcan una serie de factores como son: el lugar de hallazgo, el contenedor de las monedas, cómo están distribuidas éstas, etc. (Gurt, 1994, 82-83).

-la fecha de acuñación de las monedas más recientes del conjunto, fecha que constituiría un terminus post quem para conocer el momento a partir del cual dejaron de ingresar nuevas monedas en el conjunto. El grado de desgaste que presentan las monedas, la propia composición del conjunto, etc. (Llorens, Ripollés, Doménech, 1997, 14).

En el caso que nos ocupa, ninguna de las condiciones anteriores existe, por lo que deberemos recurrir a métodos indirectos para tratar de establecerla. El contenido de una hucha se corresponde con una variante de los denominados depósitos de ahorro regular, en los que las monedas se van añadiendo durante un período de tiempo más o menos largo (Llorens, Ripollés, Doménech, 1997, 11), por lo tanto, estaríamos ante dos cronologías claramente diferenciadas:

-una primera que se correspondería con el inicio del ahorro regular y que sería posterior a la fecha de fabricación de la hucha.

-y un momento final en el que se recupera el dinero ahorrado, para lo cual se procede a la destrucción del recipiente.

Así pues, con los escasos datos que poseemos (sus restos fraccionados y un sestercio 
sin describir ni datar) y partiendo de las anteriores premisas debemos recurrir en primer lugar a la información que nos pueda suministrar el contexto arqueológico:

\section{a) Contexto arqueológico}

Los fragmentos de la hucha aparecieron durante la tercera fase de las excavaciones de urgencia que el Museo Arqueológico Municipal de Lorca llevó a cabo en el solar de la Calle Eugenio Úbeda, 12-14 de Lorca (Murcia) entre el 5 de octubre y el 17 de noviembre de 1993 (Martínez Rodríguez, Ponce García, 1999, 298329),

En el denominado corte 2 de esta tercera fase de las excavaciones se documentaron las siguientes fases de ocupación:

I: Establecimiento almohade

II: Establecimiento bajoimperial del siglo IV d.C.

III: Establecimiento bajoimperial del siglo III d.C.

IV: Establecimiento altoimperial (siglo I d.C. y primera mitad del siglo II d.C.)

$\mathrm{V}$ : Fase tardorrepublicana

VI: Fase ibérica

En la fase III, correspondiente a un establecimiento datado en el siglo III d.C., se detectó la existencia de tres ámbitos de grandes dimensiones situados de forma correlativa $y$ orientados de Este a Oeste que se denominaron como Ambientes 6, 7 y 8 respectivamente. En concreto, la hucha se halló fragmentada en el denominado Ambiente 6 que se identifica, por sus características constructivas y por dos canalizaciones embutidas que aparecen en su muro norte con una instalación hidráulica que sería amortizada en la segunda mitad del siglo III d.C. con rellenos procedentes de actividades artesanales relacionadas con la alfarería y la fabricación de vidrio. Entre estos materiales de relleno aparecieron además de los fragmentos de la hucha: una jarra fragmentada con defectos de cocción, un cuenco completo, desechos de vidrio (filamentos, lágrimas, fragmentos de cuello, fondo, etc) así como dos monedas: un sestercio del siglo III d.C. y un radiado de Tétrico II (273-274 d.C.). En el denominado Ambiente 7 de claro uso doméstico y que aparece adosado por el Este al ambiente 6 aparecieron los siguientes materiales: TS Africana de diferentes tipologías con una cronología que abarcaria desde la segunda mitad del siglo II d.C. hasta el primer cuarto del s. IV d.C. y un radiado de Claudio II (268-270 d.C.). Por su parte, el denominado Ambiente 8, adosado al Este del Ambiente 7, presenta una gran escasez de material cerámico por lo que sus excavadores lo relacionan con un uso de establo o corral.

La aparición de sus restos en un vertedero de materiales, que rellenan un espacio ya amortizado anteriormente, hace que su datación cronológica en base a los materiales hallados sea cuanto menos complicada, puesto que la aportación de los desechos presentes en el vertedero se habría realizado durante un lapso de tiempo que no podemos cuantificar, por lo que los materiales presentes en este ámbito deben presentar una horquilla cronológica que puede ser bastante amplia, lo que se une a su propia tipología que no permite realizar ninguna precisión cronológica fiable.

Estas circunstancias nos conducen a intentar su datación en base a las monedas que aparecen entre los materiales de desecho:

\section{b) Contexto numismático}

El tipo de monedas halladas junto a los fragmentos de la hucha implican también, cronológicamente hablando, serios problemas de datación, puesto que la presencia de una moneda de Tétrico II nos llevaria a un momento posterior a la fecha de su acuñación (273-74 d.C.), pero la circulación de estas acuñaciones llega y sobrepasa perfectamente toda la primera mitad del s. IV. d.C. Un caso similar es el que presenta el radiado de Claudio II hallado en el contiguo Ámbito 8. Por otra parte, la presencia de un sestercio del s. Ill d.C., sin adscribir a un determinado emperador, así como la del sestercio que se halla incrustado en la misma pieza parecen sugerir una datación que podría llegar hasta el tercer cuarto del s. III d.C. habida cuenta del período de circulación de este tipo de monedas en la Península lbérica ${ }^{3}$.

Ante esta cronología tan laxa, podemos afinar un poco más si volvemos al hipotético contenido del recipiente: de él sólo conocemos un sestercio, que ni siquiera ha podido ser descrito, pero si cruzamos este dato con los datos

\footnotetext{
${ }^{3}$ Sobre la circulación de la moneda de bronce en el Occidente se puede consultar: Callu, 1969, 111-146; Turcan, 1963 (Para la primera mitad del s. lll d.C.). Para los depósitos monetarios de bronce: Buttrey, 1972, 33-58 y para la circulación en la Peninsula lbérica: Sagredo San Eustaquio, 1983, 173-222; Bost, Campo, Gurt, 1980, 174-202; Bost, Chaves, Depeyrot, Hiernard, Richard, 1987, 69-74; Pereira, Bost, Hiernard, 1974, 226-230; Bost, Campo, Colls, Guerrero, Mayet, 1992, 98-116.
} 


\begin{tabular}{|c|c|c|c|c|c|c|c|c|c|}
\hline Depósito & $\begin{array}{c}\text { Total } \\
\text { Monedas }\end{array}$ & Rad & Den & Ses & As & Dup & $\begin{array}{l}\text { Moneda } \\
\text { Final }\end{array}$ & Contenedor & Lugar de hallazgo \\
\hline Cabrera III & 967 & 2 & & 965 & & & 256-7 d.C. & ánfora Dressel 23 & Pecio \\
\hline Vilauba & 47 & 2 & & 44 & 1 & & 259 d.C. & $\begin{array}{l}\text { olla de cerámica } \\
\text { común }\end{array}$ & Villa \\
\hline D’Eula & 62 & 7 & & 55 & & & 259 d.C. & $\begin{array}{l}\text { vaso de cerámica } \\
\text { común }\end{array}$ & Paraje campestre \\
\hline Mas d'Aragó & 53 & 38 & & 15 & & & 266 d.C. & bolsa de tela & $\begin{array}{l}\text { Recinto termal de una } \\
\text { villa }\end{array}$ \\
\hline Bascuñuelos & 10 & 1 & & 8 & 1 & & 285 d.C. & $i ?$ & Cerca de un arroyo \\
\hline Montalegre & 994 & $i ?$ & & ¿? & & & 285 d.C.? & vaso de bronce & $\begin{array}{l}\text { Cercanias del Castillo } \\
\text { de Montalegre }\end{array}$ \\
\hline Valbeirô & 5 & 4 & & & 1 & & $\begin{array}{l}\text { post. } 270 \\
\text { d.C. }\end{array}$ & plato cerámico & Necrópolis \\
\hline O Castelo & 6 & 2 & & 1 & 2 & 1 & 270 d.C. & sin recipiente & $\begin{array}{l}\text { Camino de acceso a un } \\
\text { castro }\end{array}$ \\
\hline Clunia-2 & 24 & 18 & 1 & 1 & 4 & & 280 d.C. & sin recipiente & Casa Urbana = Clunia- 3 \\
\hline Clunia-3 & 54 & 50 & & 2 & 2 & & 274 d.C. & sin recipiente & Casa Urbana = Clunia-2 \\
\hline Pontevedra & ¿? & & 5 & & 1 & & 264 d.C. & cista de piedras & ¿? \\
\hline
\end{tabular}

Figura 3.

arqueológicos y numismáticos anteriores parece muy factible que la hucha contuviera tanto sestercios como radiados durante su periodo de vida útil $y$, por tanto, de la comparación con los depósitos peninsulares compuestos por radiados y sestercios podríamos obtener alguna información que nos ayude a determinar su cronología.

Hasta el momento 11 son los conjuntos peninsulares que presentan radiados y monedas de bronce en su composición: Pecio "Cabrera III" (Puerto de Cabrera, Baleares) ${ }^{4}$, Vilauba (Camós, Gerona) ${ }^{5}$, D’Eula (Crevillente, Alicante) ${ }^{6}$, Mas d'Aragó (Cervera del Maestrat, Castellón ${ }^{7}$, Bascuñuelos (Valle de Tobalina, Burgos) ${ }^{8}$, Montalegre (Concelho de Montalegre, Distrito de Vila Real, Portugal) ${ }^{9}$, Necrópolis de Valbeirô (Freguesia de Santa María de Sardoura, Concelho de Castelo de Paiva, Portugal) ${ }^{10}$, Poblado de O Castelo (As Laias, Cenlle, Ourense) ${ }^{11}$, Clunia-2 (Coruña del Conde, Burgos) ${ }^{12}$, Clunia-3 (Coruña del Conde, Burgos) ${ }^{13}$ y Provincia de Pontevedra ${ }^{14}$ (Fig. 3)

Del cuadro sinóptico anterior se desprende que la moneda de bronce y, en especial, los sestercios circulan (muy posiblemente de forma residual) todavía en el $280 / 85$ d.C., aunque conforme avanza el siglo y a partir del 260 d.C. su número decrece frente al de los radiados, lo cual es perfectamente lógico puesto que es en estos momentos cuando se dejan de acuñar regularmente.

Si utilizamos los conjuntos más cercanos geográficamente: Vilauba, D’Eula y Mas
d'Aragó, vemos que para fechas en torno al 270 d.C..$^{15}$ todavía existe una gran proporción de sestercios en la circulación de la fachada mediterránea. Sin embargo, hemos constatado también que en los conjuntos compuestos exclusivamente por radiados con moneda final a partir de Aureliano sus monedas más anti-

${ }^{4}$ Bost, Campo, Colls, Guerrero, Mayet, 1992; Martínez Mira, 1995-97, 129-130, n²7

5 Roure, Tremoleda, Castanyer, 1988-1989, 268-281; Martínez Mira, 1995-97, 130-131, n 28.

${ }^{6}$ González Prats, Abascal Palazón, 1987, 183-196, Martínez Mira, 1995-97, 134-135, n 46.

${ }^{7}$ Gozalbes, 1996, 383-404; Martínez Mira, 2000-2001, 298$299, n^{\circ} 41^{*}$.

${ }^{8}$ Cadiñanos Bardeci, 1995, 98-99.

${ }^{9}$ Espigueiro. Central de informaçôes regionais (http: // www.espigueiro.pt) noticia del día 12-07-2003.

${ }^{10}$ Tavares Dias 1993-94, 117-118.

${ }^{11}$ Cepas, Álvarez, López, 1999, 147-158.

${ }^{12}$ Gurt, 1985, 133-144, Martínez Mira, 1995-1997, 144, nº 79.

${ }^{13}$ Gurt, $1985,133-145$.

${ }^{14}$ Cavada Nieto, 1984, 181-191, Martínez Mira, 1995-1997, $138, n^{\circ} 56$.

${ }^{15}$ Hemos de anotar que para la cronología final del conjunto de Vilauba se debe tomar como referencia un radiado de Claudio II acuñado en 269-270 d.C. que se halló en el mismo estrato de derrumbe aunque en una habitación diferente (Castanyer, Tremoleda, 1999, 320 y 326-330). Hemos de notar la similitud con el radiado de Claudio II hallado en el Ámbito 8 contiguo a la zona en donde se hallaron los restos de la hucha 
guas pertenecen a Valeriano/Galieno y se acuñan en torno al 260 d.C. (Martínez Mira, 1995$1997,152)$ lo que indicaría una rápida desaparición del sestercio y de los radiados acuñados entre Gordiano III y Valeriano en este tercer cuarto del s. III d.C. Aunque existen ejemplos del mantenimiento residual dentro de los circuitos monetarios de estos tipos de monedas en momentos bastante posteriores (Abascal Palazón, 1994, 143-158).

Por tanto, si unimos los datos que se desprenden del cuadro anterior con los datos de las excavaciones creemos que podemos situar perfectamente la cronología del uso y destrucción de la hucha en torno al tercer cuarto del s. III d.C. y que podría representar para la comarca de Lorca el final de la circulación de las monedas de bronce en este territorio, al respecto, hemos de constatar la presencia de dos conjuntos monetarios del s. III d.C. hallados en su término municipal y que podrian por su composición representar los momentos iniciales y finales en este proceso de sustitución de los sestercios por los radiados para este ámbito geográfico concreto:

-Depósito del Aljibe de Poveda. Este depósito en su origen tendría una treintena de monedas todas sestercios, con un arco cronológico que abarcaría el s. I al III d.C. y cuya última acuñación conocida perteneceria a Gordiano III. (Martínez Rodríguez, 1995, 216 y 222, Martínez Mira, 2000-2001, 300, n095).

-Cerro de la Encantada. Sus 205 monedas son exclusivamente radiados y abarcan desde Valeriano I hasta Probo (este depósito no incluye ninguna moneda de Tétrico II). (Gomis Justo, 1990, 101-110; Martínez Mira, 2000-2001, 305, $n^{\circ} 110$ ).

\section{CONCLUSIONES:}

Tipológicamente pensamos que el ejemplar del Museo Arqueológico Municipal de Lorca se puede clasificar dentro del grupo II de Graeven, aunque, formalmente, no presente la forma de una hucha convencional como las que se describen en este grupo. Al ser cerámicas de producción local, la variabilidad en su forma es bastante alta con adaptaciones que presumiblemente no se den nada más que en las piezas conservadas, un ejemplo de ello lo podemos observar en la hucha hallada en Wiesloch que presenta en su base inferior una serie de agujeros para permitir que pase el aire y se desaloje la humedad del interior de la hucha. Humedad que afectaría a las monedas oxidándolas si estas permanecieran largo tiempo en el interior de la hucha (Hensen, 1998, 6 y Fig. 1). Estaríamos pues ante una de estas adaptaciones del tipo inicial con las siguientes características:

\section{Comunes:}

-Cerámica común de producción local tosca

-Ausencia de decoración

-Cuerpo central en donde se alojan las monedas de forma globular (tipo olla)

-Su volumen interno útil para contener monedas, unos $1.500 \mathrm{ml}$., es similar al de las huchas descritas de este tipo.

\section{Diferentes:}

-No presenta una forma de hucha propiamente dicha, sino que es una adaptación de una forma de cerámica de uso doméstico común a las funciones de hucha al realizarle una ranura para la inserción de las monedas y aprovechar al mismo tiempo la estrechez de su cuello que impide la salida de las monedas desde el interior. Esta última característica contrasta con los casos de algunas huchas halladas en Britania, en donde Robertson constata que existen: "A few flagons or jars in fact had their necks stopped up and a slot cut in the side for the insertion of coins, after the manner of a modern "piggy-bank"" (Robertson, 1988, 24), es decir que se les tapona el cuello (se les modifica una parte de su forma) para evitar la salida de las monedas.

-Un elemento diferenciador importante sería la ubicación de la ranura de inserción de las monedas, que en lugar de situarse claramente visible en la zona más alta del cuerpo globular de la hucha se sitúa en la base del pie de manera que su existencia pasa totalmente desapercibida. Como modelo más cercano tendríamos la hucha que contenía el depósito monetario hallado en 1910 en Nottingham (Grueber, 1910, 205-206) en donde "one of two pots (one inverted inside the other) had a slot out in its bottom" (White, 1981, 305, nota 126), aunque en este caso la ranura seguiría estando claramente visible.

A partir de los datos anteriores y dado que apareció en una zona con restos de actividades artesanales podemos intuir que muy probablemente el alfarero que la realizó y que tendría su sede en las cercanías, sería su propietario puesto que lo que parece transmitir con 
mas fuerza esta pieza es el deseo de ocultación, de pasar desapercibida con que se realizó la hucha y si fuera una pieza que se hubiera realizado en serie o incluso por encargo perdería parte de estas características. Este deseo se ve reforzado cuando observamos la dificultad creciente que presenta la acción de insertar una nueva moneda en la hucha. Cada vez que se desea realizar esta operación hay que voltearla y cada nueva moneda que se inserta representa unos gramos más de peso que dificultan esta operación.

Precisamente, este movimiento necesario para la inserción de una nueva moneda y la presión generada por las que ya estaban en el interior de la hucha sobre el orificio del arranque interno del cuello de la jarrita al realizarlo, serían los responsables de que una de las monedas quedará encajada y llegara hasta nosotros.

En cuanto a la cantidad de monedas que pudo contener es imposible saberlo aunque no creemos que fuera muy importante dado su volumen útil. Estamos previsiblemente ante una economía débil, un poco superior a la de subsistencia lo que le permitiría poder destinar regularmente una serie de monedas al ahorro. El dinero que se introduce en la hucha es el dinero que se ahorra y que no es indispensable para el desarrollo de la vida cotidiana, podríamos decir pues que estamos ante la renta disponible de su poseedor en un momento determinado. Por otra parte, el uso de este tipo de recipientes para guardar monedas implica también el propósito de no disponer de ellas a menos que exista un caso de necesidad, haya suficiente dinero guardado para el fin que inicialmente se perseguía o bien que la hucha se haya llenado, en cualquiera de estos casos disponer del dinero acumulado implica siempre la destrucción del contenedor y su no reutilización posterior. Estamos pues ante una finalidad diferente a la que se nos presenta cuando el dinero se guarda en un vaso o plato normal, puesto que estos casos se puede disponer del dinero en cualquier momento sin tener que destruir el recipiente.

Por último, el yacimiento arqueológico en donde se halló la pieza se identifica tradicionalmente con la mansio de la Via Augusta Eliocroca o Eliocrora, mencionada en el Itinerario de Antonino (401.6) en el tramo entre Carthago Nova y Basti (Amante Sánchez, Cepas Palanca, 172) hallándose dentro del área de influencia económica de Carthago Nova. Para el s. III d.C. se ha constatado en la zona de Lorca una gran estabilidad en cuanto al número de explotaciones agrarias detectadas en su territorio, se mantienen 25 de los establecimientos documentados en el s. II d.C. surgiendo otros nuevos (Martínez Rodríguez, $1995,214-216$ y 222) en contraste con el panorama de crisis que parece vivir Carthago Nova y su hinterland a partir del s.Il d.C. y que se ha puesto en relación con el cese de sus explotaciones mineras y la bajada de producción de sus salinas y salazones (Murcia Muñoz, 1997, 221-227). Muy posiblemente este panorama de crisis cambie cuando se publiquen los resultados de las últimas excavaciones de esta zona. La hucha sería el testigo de un cierto nivel de transacciones económicas y al mismo tiempo también del movimiento monetario de la zona dado que estamos en un nudo de comunicaciones y en una zona artesanal, quizá no muy potente habida cuenta del volumen de restos hallados en el vertedero y que estaría de acuerdo con un panorama de estabilidad en la zona al menos, para el tercer cuarto del s. III d.C., estaríamos pues ante un testigo de la normalidad en esta zona en un período en el que según la historiografía peninsular se producen una serie invasiones, crisis, etc, que afectarian también a esta zona ${ }^{16}$

Isidro Martínez Mira
Dpto. de Química Inorgánica
Facultad de Ciencias
Universidad de Alicante
Apdo.99
03080Alicante
isidro@ua.es

\section{BIBLIOGRAFÍA}

ABASCAL PALAZÓN, J.M., 1994: "Hallazgos arqueológicos y circulación monetaria. Disfunciones metodológicas en el estudio de la Hispania romana", IX Congreso Nacional de Numismática, 143-158, Elche.

AMANTE SÁNCHEZ, M., CEPAS PALANCA, A., 2000 "Eliocrora", TIR, J-30, 172, Madrid.

BOST, J.-P., CAMPO, M., COLLS, D., GUERRERO, V., MAYET, F., 1992: L'Épave Cabrera III (Majorque). Échanges commerciaux et circuits monétaires au milieu du llle siècle après Jésus-Christ, París.

BOST, J.-P., CAMPO, M., GURT, J.M., 1979: "La circulación monetaria en Hispania durante el período RomanoImperial: Problemática y conclusiones generales", I Symposium Numismático de Barcelona, T. II, 174-202, Barcelona.

BOST, J.-P., CHAVES, F., DEPEYROT, G., HIERNARD, J., RICHARD, J.-C., 1987: Belo IV: Les Monnaies, Madrid.

\footnotetext{
${ }^{16}$ Sobre historiografia y la bibliografía del tema de las invasiones se puede consultar: Peña Cervantes, 2000, 469-492, Martínez Mira, 1992.
} 
BUTTREY, T.V., 1972: "A hoard of sestertii from Bordeaux and the problem of bronze circulation in the $3^{\text {rd }}$ Century A.D.", American Numismatic Society Museum Notes, 18 , 33-58.

CADIÑANOS BARDECI, I., 1995: El Valle de Tobalina, Excmo. Ayto. del Valle de Tobalina, $2^{\mathrm{a}}$ Ed.

CALLU, J.-P., 1969: La Politique monétaire des Empereurs Romains de 238 à 311 , París.

CASTANYER, P., TREMOLEDA, J., 1999: La villa romana de Vilauba. Un exemple de l'ocupació i explotació romana del territori a la comarca del Pla de l'Estany, Girona.

CAVADA NIETO, M., 1984: "Dos tesorillos monetarios aparecidos en Galicia", // Congreso Galaico-Minhoto, 181191.

CEPAS, A., ÁLVAREZ, Y., LÓPEZ, L. F., 1999: "Circulación monetaria en zonas mineras: El poblado de O Castelo (Orense)", en Centeno, R.M.S., García-Bellido, M. P., Mora, G., (Coords.): Actas // Encuentro Peninsular de Numismática Antigua, 1997, Anejos de Archivo Español de Arqueologia, XX, 147-158, Porto.

CHEILIK, S. M., 1963: "A Roman Terracotta Savings Bank", American Journal of Archaeology, Vol. 67, $\mathrm{n}^{\circ} 1,70-71$.

DUKAT, Z., SARIC, I., 1995-96: "Rimska stedna kasica iz Siska", Vjesnik Archeoloskog Muzeja u Zagrebu, s.3, 2829, 97-110.

ESPIGUEIRO: Central de informaçôes regionais (http: // www.espigueiro.pt) noticia del dia 12-07-2003, depósito de Montalegre (Portugal).

GOMIS JUSTO, M., 1990: "Las monedas de la Colección L. Tormo (Sagunto)", Gaceta Numismática, 97-98, 101-110.

GONZÁLEZ PRATS, A., ABASCAL PALAZÓN, J.M., 1987: "La ocultación monetal de la D'Eula, Crevillente (Alicante) y su significación para el estudio de las invasiones del siglo III", Lucentum, 6, 183-196.

GOZALBES, M., 1996: "El tesoro del Mas d'Aragó (Cervera del Maestre, Castellón) y la década del 260 d.C.", Cuadernos de Arqueología y Prehistoria Castellonenses, 17, 383-404.

GRAEVEN, H., 1901: "Die thönerne Sparbüchse im Altertum", Jahrbuch des Kaiserlichen Deutschen Archäologischen Instituts, 16, 160-189.

GRUEBER, H.A., 1910: "A Find of Roman Coins at Nottingham", Numismatic Chronicle, X, 4 th ser., 205-206.

GURT, J.M., 1985: Clunia III. Hallazgos monetarios. La romanización de la Meseta Norte a través de la circulación monetaria en la ciudad de Clunia, EAE 145, Madrid.

GURT, J.M., 1994: "Podem parlar d'atresorament durant el segle III a I'Imperi Romà?", Tresors del Món Antic, VII Cicle de Conferencies, Gabinet Numismàtic de Catalunya, 81-90, Barcelona.

HENSEN, A., 1998: "Eine Römische Sparbüchse aus Wiesloch", Archäologische Nachrichten aus Baden, 59, 3-6.

HUSSONG, L., CÜPPERS, H., 1972: "Die Trierer Kaiserthermen. Die spätrömische und frühmittelalterliche Keramik". Trierer Grabungen und Forschungen I, 2, Mainz.

LLORENS, Ma. M., RIPOLLÉS, P.P., DOMÉNECH, C., 1997: Monedes d'ahir, tresors de hui, Valencia.

MARTÍNEZ MIRA, 1., 1992: Fuentes para el estudio de las supuestas invasiones del siglo III d.C. en la Península Ibérica, Tesina de licenciatura inédita, Universidad de Alicante.

MARTÍNEZ MIRA, 1., 1995-1997: "Tesorillos del S. III d.C. en la Peninsula Ibérica", Lucentum, XIV-XVI, 119-180.

MARTÍNEZ MIRA, I., 2000-2001: "Tesorillos del S. III d.C. en la Península Ibérica (II)", Lucentum, XIX-XX, 297-307. MARTÍNEZ RODRÍGUEZ, M., 1995: "EI poblamiento rural romano en Lorca", en Noguera, J.M. (Coord.): Poblamiento rural romano en el Sureste de Hispania, 203225, Murcia.

MARTÍNEZ RODRÍGUEZ, M., PONCE GARCÍA, J., 1999: "Excavaciones arqueológicas de urgencia en la Calle Eugenio Úbeda. 12-14 (Lorca, Murcia)", Memorias de Arqueologia, 8, 298-329.

MURCIA MUÑOZ, A. J., 1997: "Poblamiento rural romano en el campo de Cartagena: el tránsito de los siglos II al III d.C.", XXIV Congreso nacional de Arqueologia, 221-226, Cartagena.

PEÑA CERVANTES, Y., 2000 : "La «crisis» del siglo III en la historiografía española", Espacio, Tiempo y Forma, Serie Il, Historia Antigua, 13, 469-492.

PEREIRA, I., BOST, J.-P., HIERNARD, J., 1974: Fouilles de Conimbriga III. Les Monnaies, París.

REALACADEMIA ESPAÑOLA, 1998: Diccionario de la Lengua Española, $21^{\text {a }}$ Edición, 2 Vol., Madrid.

ROBERTSON, A.S., 1988: "Romano-British coin hoards: their numismatic, archaeological and historical signifiance", en Casey, P.J., Reece, R., 1988: Coins and the Archaeologist, 13-38, London. = British Archaeological Reports, 4, 1974.

ROBINSON, D.M., 1924: "Some Roman Terra-Cotta SavingsBanks", American Journal of Archaeology, Vol. 28, $n^{\circ} 3$, 239-250.

ROURE, A., TREMOLEDA, J., CASTANYER, P., 1988-1989. "Troballa d'un conjunt monetari a Vilauba (Camós, Pla de l'Estany)", Empúries, 48-50, vol. 11, 268-281.

SAGREDO SAN EUSTAQUIO, L., 1983: "La circulación y desaparición del bronce en la Hispania Romana (193285)", Estudios en Homenaje a Don Claudio Sánchez Albornoz, Vol. 1, 173-222.

TAVARES DIAS, L.A., 1993-1994: "Necrópoles do territorium de Tongobriga", Conimbriga, 32-33, 107-136 + 12 lám.

THÉDENAT, H., 1969: "Loculus, loculi, locellus, loculamenta", en Mm. Ch. Daremberg, Edm. Saglio, Edm. Pottier, 1969: Dictionnaire des Antiquites Grecques et Romaines, T. III/ 2, L-M, 1292-1295, Reimpresión, Graz.

TURCAN, R., 1963: Le trésor de Guelma, París.

WHITE, A.J., 1981: "A Roman Pottery Money-Box from Lincoln", Britannia, 12, 302-305. 